Research

Open Access

\title{
Burn size determines the inflammatory and hypermetabolic response
}

\author{
Marc G Jeschke ${ }^{1,2}$, Ronald P Mlcak ${ }^{1}$, Celeste C Finnerty ${ }^{1,2}$, William B Norbury ${ }^{1}$, \\ Gerd G Gauglitz ${ }^{1,2}$, Gabriela A Kulp ${ }^{1}$ and David N Herndon ${ }^{1,2}$
}

\author{
1Shriners Hospitals for Children, 815 Market Street, Galveston, TX 77550, USA \\ 2Department of Surgery, University Texas Medical Branch, Galveston, TX, 77550 USA
}

Corresponding author: Marc G Jeschke, majeschk@utmb.edu

Received: 31 Jan 2007 Revisions requested: 2 Mar 2007 Revisions received: 20 Apr 2007 Accepted: 23 Aug 2007 Published: 23 Aug 2007

Critical Care 2007, 11:R90 (doi:10.1186/cc6102)

This article is online at: http://ccforum.com/content/11/4/R90

(C) 2007 Jeschke et al.; licensee BioMed Central Ltd.

This is an open access article distributed under the terms of the Creative Commons Attribution License (http://creativecommons.org/licenses/by/2.0), which permits unrestricted use, distribution, and reproduction in any medium, provided the original work is properly cited.

\begin{abstract}
Background Increased burn size leads to increased mortality of burned patients. Whether mortality is due to inflammation, hypermetabolism or other pathophysiologic contributing factors is not entirely determined. The purpose of the present study was to determine in a large prospective clinical trial whether different burn sizes are associated with differences in inflammation, body composition, protein synthesis, or organ function.
\end{abstract}

Methods Pediatric burned patients were divided into four burn size groups: $<40 \%$ total body surface area (TBSA) burn, $40-$ $59 \%$ TBSA burn, $60-79 \%$ TBSA burn, and $>80 \%$ TBSA burn. Demographic and clinical data, hypermetabolism, the inflammatory response, body composition, the muscle protein net balance, serum and urine hormones and proteins, and cardiac function and changes in liver size were determined.

Results One hundred and eighty-nine pediatric patients of similar age and gender distribution were included in the study ( $<40 \%$ TBSA burn, $n=43 ; 40-59 \%$ TBSA burn, $n=79 ; 60-$ $79 \%$ TBSA burn, $n=46 ;>80 \%$ TBSA burn, $n=21$ ). Patients with larger burns had more operations, a greater incidence of infections and sepsis, and higher mortality rates compared with the other groups $(P<0.05)$. The percentage predicted resting energy expenditure was highest in the $>80 \%$ TBSA group, followed by the 60-79\% TBSA burn group $(P<0.05)$. Children with $>80 \%$ burns lost the most body weight, lean body mass, muscle protein and bone mineral content $(P<0.05)$. The urine cortisol concentration was highest in the $80-99 \%$ and $60-79 \%$ TBSA burn groups, associated with significant myocardial depression and increased change in liver size $(P<0.05)$. The cytokine profile showed distinct differences in expression of IL8, TNF, IL-6, IL-12p70, monocyte chemoattractant protein-1 and granulocyte-macrophage colony-stimulating factor $(P<0.05)$.

Conclusion Morbidity and mortality in burned patients is burn size dependent, starts at a $60 \%$ TBSA burn and is due to an increased hypermetabolic and inflammatory reaction, along with impaired cardiac function.

\section{Introduction}

The stress response to burn injury is similar to severe trauma or critical care but differs in its severity and duration. The inflammatory response starts immediately after trauma and persists for almost 5 weeks postburn [1]. The hypermetabolic response after a major burn is characterized by a hyperdynamic response with increased body temperature, increased oxygen and glucose consumption, increased $\mathrm{CO}_{2}$ production, increased glycogenolysis, increased proteolysis, increased lipolysis, and increased futile substrate cycling [2]. This response begins on the fifth day postinjury and continues up to 24 months postburn, causing the loss of lean body mass, the loss of bone density, muscle weakness, and poor wound healing [2-4]. The increased metabolic requirements cause tissue catabolism, leading to nitrogen loss and a potentially lethal depletion of essential protein stores [5]. The energy requirements are met by the mobilization of proteins and amino acids. Increased protein turnover, degradation and negative nitrogen balance are all characteristic of this severe critical illness [2,5]. As a consequence, the structure and function of essential 
organs, such as the heart, the liver, skeletal muscle, the skin, the immune system and cellular membrane transport functions, are compromised [6-8].

Numerous studies show that an increased burn size leads to increased mortality of burned patients $[9,10]$. Furthermore, it was speculated that the burn size determines the extent of the hypermetabolic response. Whether mortality is due to inflammation, hypermetabolism or other pathophysiologic contributing factors is not entirely determined. The purpose of the present study was to determine in a large prospective clinical trial whether different burn sizes are associated with differences in inflammation, in body composition, in protein synthesis, and in organ function.

\section{Patients and methods Participants}

All thermally injured children over a time period of 9 years who were admitted to our burns unit and required at least one surgical intervention were included in the study. Patients were resuscitated according to the Galveston formula using Ringer's lactate. Within 48 hours of admission all patients underwent total burn wound excision, in which the wounds were covered with the available autograft skin and an allograft was used to cover any remaining open areas. After the first operative procedure it was 5-10 days until the donor site was healed, and patients were then taken back to the operating theater. The skin graft procedure was repeated until all open wound areas were covered with autologous skin material.

All patients underwent the same nutritional treatment to a standardized protocol. We used the Galveston formulas Galveston Infant, Galveston Revised, and Galveston Adolescent. The formula changes with age based on the body surface alterations that occur with growth. The intake is approximately calculated as $1,500 \mathrm{kcal} / \mathrm{m}^{2}$ body surface area $+1,500 \mathrm{kcal} / \mathrm{m}^{2}$ area burn. The composition of the nutritional supplement is also important. The optimal dietary composition contains 1-2 g/kg/day protein, which provides a calorie-tonitrogen ratio of approximately 100:1 with the suggested caloric intakes. Nonprotein calories can be given either as carbohydrate or as fat, with clinical advantages for the carbohydrates. The diet was delivered by enteral nutrition, if possible, in all our patients. Total parenteral nutrition was only used as a supplemental form of nutrition when the calculated intake could not be achieved.

Patient demographics (age, date of burn and admission, sex, burn size and depth of burn) and concomitant injuries, such as inhalation injury, sepsis, morbidity and mortality, were recorded. Sepsis was defined as a blood culture identifying the pathogen during hospitalization or at autopsy, in combination with leucocytosis or leucopenia, hyperthermia or hypothermia, and tachycardia. Wound healing was evaluated from the time of donor site healing, and therefore from the time between operative interventions.

\section{Indirect calorimetry}

As part of our routine clinical practice, all patients underwent resting energy expenditure (REE) measurements within 1 week following hospital admission, at 2-4 weeks after hospital admission, at discharge, and at 6 months postburn. Measurements of REE were performed between midnight and 5:00 am while the patients were asleep and receiving continuous feeding. The REE was measured using a Sensor-Medics Vmax 29 metabolic cart (Sensor-Medics, Yorba Linda, CA, USA). Subjects were tested in a supine position while under a large, clear, ventilated hood. The REE was calculated from the oxygen consumption and carbon dioxide production using equations described by Weir [11] All REE measurements were made at ambient temperatures of $30^{\circ} \mathrm{C}$, which is the standard environmental setting for all patient rooms in our acute burn intensive care unit.

The REE measurements were used to guide nutritional management and to assess the level of metabolism. The discharge REE measurement was used to determine the level of hypermetabolism when the burn wounds were 95\% healed and was included as part of the study. Measured values were compared with predicted norms based upon the Harris-Benedict equation [12]. The REE studies were repeated at 6,9 and 12 months postburn when the patients returned for outpatient surgery. Assessments of the REE at these time points were completed utilizing the methodology and environmental settings as described above. For statistical comparison, energy expenditure was expressed both as the absolute REE and as the percentage of the basal metabolic rate predicted by the Harris-Benedict equation.

\section{Muscle protein net balance}

The muscle protein net balance was calculated from the product of the amino acid concentration difference and the blood flow, as previously published [13-16].

\section{Body composition}

The height and the body weight were determined clinically 5 days after admission and at discharge. The total body lean mass, fat, bone mineral density, and bone mineral content were measured by dual-energy X-ray absorptiometry. The Hologic model QDR-4500W DEXA (Hologic Inc., Waltham, MA, USA) was used to measure the body composition. To minimize systematic deviations, the Hologic system was calibrated daily against a spinal phantom in the anteroposterior, lateral, and single-beam modes. Individual pixels were calibrated against a tissue bar phantom to determine whether the pixel was reading bone, fat, lean tissue, or air. Plain anteriorposterior and lateral tibia-fibula X-rays were taken of each subject at each follow-up period to evaluate for possible 
premature closure of epiphyseal plates induced by anabolic agents.

\section{Serum hormones, proteins, and cytokines}

Blood or urine was collected from the burn patients at the time of admission, preoperatively, and 5 days postoperatively for 4 weeks for serum hormone, protein, cytokine and urine hormone analysis. Blood was drawn in a serum-separator collection tube (BD, Franklin Lakes, NJ, USA) and was centrifuged for 10 minutes at $1,300 \times g$. The serum was then removed and stored at $-70^{\circ} \mathrm{C}$ until assayed.

Serum hormones and acute phase proteins were determined using high-performance liquid chromatography and ELISA techniques. The Bio-Plex Human Cytokine 17-Plex panel was used with the Bio-Plex Suspension Array System (Bio-Rad, Hercules, CA, USA) to profile expression of 17 inflammatory mediators: IL-1 $\beta$, IL-2, IL-4, IL-5, IL-6, IL-7, IL-8, IL-10, IL12p70, IL-13, IL-17, granulocyte colony-stimulating factor, granulocyte-macrophage colony-stimulating factor, IFN $\gamma$, monocyte chemoattractant protein-1, macrophage inflammatory protein-1 $\beta$, and TNF. The assay was performed according to the manufacturer's instructions. Briefly, serum samples were thawed and then centrifuged at $1,300 \times g$ for 3 minutes at $4^{\circ} \mathrm{C}$. Serum samples were then incubated with microbeads labeled with specific antibodies to one of the aforementioned cytokines for 30 minutes. Following a washing step, the beads were incubated with the detection antibody cocktail (each antibody specific to a single cytokine). After another washing step, the beads were incubated with streptavidin-phycoerythrin for 10 minutes, were washed again, and the concentrations of each cytokine were then determined using the array reader.

Urine cortisol was determined by standard laboratory techniques, measuring for the urine amount, creatinine and creatinine clearance.

\section{Liver and cardiac changes}

Ultrasound measurements in this study were made with the HP Sonos 100 CF echocardiogram (Hewlett Packard Imaging Systems, Andover, MA, USA). To obtain the ultrasound liver weight, a $3.5 \mathrm{MHz}$ transducer was placed directly below the midline of the rib cage on the right upper quadrant on a vertical line running through the right nipple with the patient in the supine position. Once the liver was visualized, measurements were made by scanning in a plane perpendicular to the base of the liver. The base of the liver, as well as the free edge hepatic dome, was marked on the display screen and the distance between these two points was automatically measured.

The formula used for estimating the liver weight from the single longitudinal scan along the right nipple line was weight $=$ $(1.15 /)^{3} d$, where $\beta^{\beta}$ represents the volume of a cube cut in half diagonally to visualize the approximate shape of the normal liver in situ. A factor of 1.15 was used to correct for the portion of the liver (15\%) lateral to the left nipple line and representing the most inferior point of the liver. This correction was estimated from the liver at autopsy. The density $(d)$ of the liver was measured on several sections by water displacement. Determining the right nipple line was not problematic unless the nipple was obliterated by a severe burn to the thorax. In these cases an approximation was made and recorded as such. The actual size was then compared with the predicted size.

M-mode echocardiograms were completed as follows. At the time of the study, none of the patients presented with or previously suffered from other concomitant diseases affecting cardiac function, such as diabetes mellitus, coronary artery disease, longstanding hypertension, or hyperthyroidism. The study variables included the resting cardiac output, the cardiac index, the stroke volume, the resting heart rate and the left ventricular ejection fraction. The stroke volume and cardiac output were adjusted for body surface area and were expressed as indexes. All ultrasound measurements were made with the Sonosite Titan echocardiogram, with a $3.5 \mathrm{MHz}$ transducer. Recordings were performed with the subjects in a supine position and breathing freely. M-mode tracings were obtained at the level of the tips of the mitral leaflets in the parasternal long axis position and measurements were performed according to the American Society of Echocardiography recommendations. Left ventricular volumes determined at end diastole and end systole were used to calculate the ejection fraction, the stroke volume, the resting cardiac output and the cardiac index. Three measurements were performed and averaged for data analysis.

\section{Ethics and statistics}

The study was reviewed and approved by the Institutional Review Board of the University Texas Medical Branch, Galveston, TX, USA. Prior to the study, each subject, parent or child's legal guardian signed a written informed consent form. Analysis of variance with post-hoc Bonferroni correction, paired and unpaired Student's $t$ tests, chi-square analysis, and Mann-Whitney tests were used. Data are expressed as the mean \pm standard deviation in the tables or as the mean \pm standard error of the mean in the figures. Significance was accepted at $P<0.05$.

\section{Results \\ Demographics}

One hundred and eighty-nine severely burned children were included in the present study. The patients' demographics are presented in Table 1. There was no significant difference in age and in the gender distribution between the different burn sizes (Table 1).

The average time from burn to hospital admission was significantly shorter in the $>80 \%$ TBSA burn group and in the $60-$ $79 \%$ TBSA burn group when compared with the other two groups $(P<0.05)$ (Table 1$)$. The length of hospital stay was 
Table 1

\begin{tabular}{|c|c|c|c|c|}
\hline & $\begin{array}{l}<40 \% \text { TBSA burn group } \\
(n=43)\end{array}$ & $\begin{array}{l}\text { 40-59\% TBSA burn group } \\
(n=79)\end{array}$ & $\begin{array}{l}60-79 \% \text { TBSA burn group } \\
(n=46)\end{array}$ & $\begin{array}{l}>80 \% \text { TBSA burn group } \\
(n=21)\end{array}$ \\
\hline Age (years) & $7.3 \pm 0.8$ & $7.9 \pm 0.8$ & $7.4 \pm 0.7$ & $8.8 \pm 1.3$ \\
\hline Gender (female/male) & $19 / 24$ & $31 / 48$ & $19 / 27$ & $7 / 14$ \\
\hline Time to admission (days) & $9 \pm 2$ & $7 \pm 1$ & $6 \pm 1^{+}$ & $3 \pm 1^{*}$ \\
\hline Length of stay (days) & $20 \pm 2$ & $26 \pm 2$ & $45 \pm 5^{+}$ & $70 \pm 12^{*}$ \\
\hline TBSA (\%) & $34 \pm 1$ & $50 \pm 1^{* *}$ & $70 \pm 1^{+}$ & $87 \pm 1^{*}$ \\
\hline Third-degree burn (\%) & $24 \pm 2$ & $36 \pm 2^{\star *}$ & $60 \pm 2^{+}$ & $75 \pm 5^{*}$ \\
\hline Length of stay/\% TBSA (days/\%) & $0.55 \pm 0.05$ & $0.53 \pm 0.03$ & $0.64 \pm 0.05^{+}$ & $0.79 \pm 0.13^{*}$ \\
\hline Operations $(n)$ & $2 \pm 0.2$ & $3 \pm 0.2$ & $6 \pm 0.5^{+}$ & $8 \pm 1^{*}$ \\
\hline Inhalation injury (\%) & 30 & 28 & 46 & $71^{*}$ \\
\hline Ventilator (\%) & 0 & 10 & $15^{+}$ & $35^{*}$ \\
\hline Infection (\%) & 5 & 18 & 15 & $19^{*}$ \\
\hline Sepsis (\%) & 0 & 5 & $24^{+}$ & $38^{*}$ \\
\hline Mortality (\%) & 0 & 0 & $13^{+}$ & $29^{*}$ \\
\hline
\end{tabular}

Data presented as the mean \pm standard deviation or percentage. TBSA, total body surface area. * Significant difference between the $>80 \%$ TBSA burn group compared with the $<40 \%$ and $40-59 \%$ TBSA burn groups $(P<0.05)$. ** ${ }^{*}$ ignificant difference between $40-59 \%$ TBSA versus $<40 \%$ TBSA burn group, $P<0.05$. ${ }^{\dagger}$ Significant difference between the $60-79 \%$ TBSA burn group compared with the $<40 \%$ and $40-59 \%$ TBSA burn groups $(P<0.05)$.

shortest in the $<40 \%$ TBSA burn group, followed by the $40-$ $59 \%$ TBSA burn group, and was longest in the large burn size groups (60-79\% and $>80 \%$ TBSA burn groups, respectively) $(P<0.05)$ (Table 1). Similarly, the length of stay divided by the burn size was longest in the $>80 \%$ TBSA burn group, followed by the $60-79 \%$ TBSA burn group $(P<0.05)$. There was no difference between the $<40 \%$ TBSA burn group and the $40-$ $59 \%$ TBSA burn group. The number of operations was greatest in the larger burn groups followed by the 60-79\% TBSA, $40-59 \%$ TBSA and $<40 \%$ TBSA required the least amount of surgeries. The $>80 \%$ TBSA burn group had significantly more patients with an inhalation injury and ventilator requirement compared with the other three groups $(P<0.05)$. Similarly, infection, sepsis and mortality was highest in the large burns group, followed by the 60-79\% TBSA burn group and then the other two smaller burn groups $(P<0.05)$.

\section{Indirect calorimetry}

The percentage predicted REE was significantly different between the four groups. Children suffering from burns of $<40 \%$ TBSA had only a slight increase in the percentage predicted REE and returned to the normal range at 6 months postburn (Figure 1). Children with 40-59\% TBSA burn and $60-79 \%$ TBSA burn had a significantly increased percentage predicted REE compared with children with $<40 \%$ TBSA burns $(P<0.05)$ (Figure 1). The highest percentage predicted REE was in children with burns of $>80 \%$ TBSA $(P<0.05)$ (Figure 1). Children with large burns demonstrated persistent elevated percentage predicted REEs at 6 months postburn (Figure 1). This indicates persistent hypermetabolism in chil- dren with burns of $>60 \%$ TBSA at least up to 6 months postburn.

\section{Peripheral muscle protein net balance}

All burned children had a negative net protein balance in skeletal muscle. The greatest muscle protein loss was found in children with $60-79 \%$ TBSA burns and $>80 \%$ TBSA burns, indicating increased hypermetabolism and catabolism in patients sustaining a burn of $>60 \%$ TBSA $(P<0.05)$ (Figure 2).

\section{Body composition}

There were distinct differences in body composition between the four burn groups. Children with $>80 \%$ TBSA burns lost the most body weight, lean body mass and bone mineral content compared with the other groups $(P<0.05)$ (Figure 3$)$. The 60-79\% TBSA burn group showed a significant loss in body weight, lean body mass, and bone mineral content that was significant compared with the $<40 \%$ and $40-59 \%$ TBSA burn groups $(P<0.05$ ) (Figure 3 ). The $<40 \%$ and $40-59 \%$ TBSA burn groups presented almost no loss of body weight, of lean body mass, and of bone mineral content (Figure 3 ).

\section{Serum hormones, proteins and cytokines}

Serum insulin growth factor-I (IGF-I) decreased immediately after burn in all four groups (Figure 4 ). In the $<40 \%$ TBSA and 40-59\% TBSA burn groups, serum IGF-I increased over time and was significantly increased compared with the 60-79\% and $>80 \%$ TBSA burn groups $21-40$ days postburn $(P<$ 0.05 ) (Figure 4). Serum insulin like growth factor binding pro- 
Figure 1

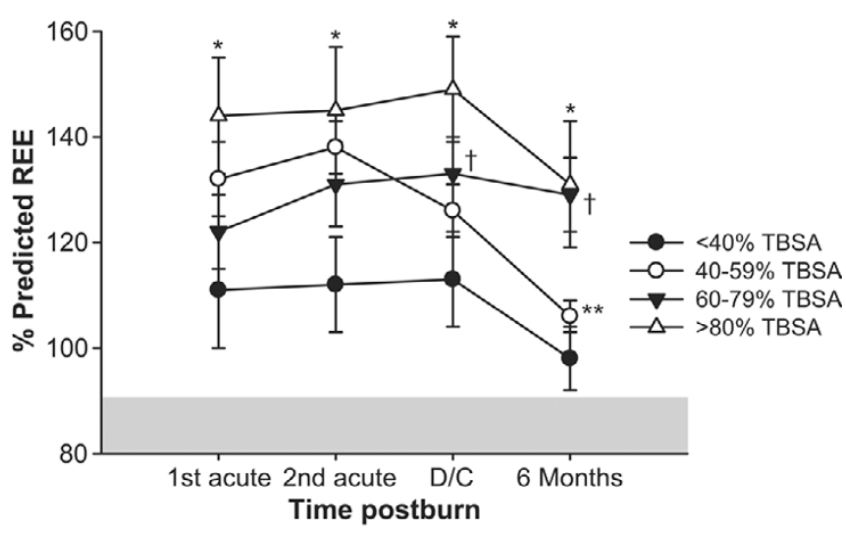

Percentage predicted resting energy expenditure. The highest percentage predicted resting energy expenditure (REE) was in children with burn $>80 \%$ of their total body surface area (TBSA), followed by $60-$ $79 \%$ TBSA burns. Children with large burns demonstrated persistent elevated percentage predicted REEs at 6 months postburn, while children with smaller burns approached the normal range. Measurements were performed at week 1 (1st acute), weeks 2-4 (2nd acute), discharge $(D / C)$, and at 6 months postburn. *Significant difference between $>80 \%$ TBSA burn group versus $<40 \%$ TBSA burn group, $P<$ 0.05 . ${ }^{*}$ Significant difference between $>80 \%$ and $60-79 \%$ TBSA burn groups versus $40-59 \%$ TBSA burn group, $P<0.05$. 'Significant difference between $60-79 \%$ TBSA burn group versus $<40 \%$ TBSA burn group, $P<0.05$.

tein-3 (IGFBP-3) decreased with a burn, but its decrease was significantly attenuated in the $<40 \%$ TBSA burn group $(P<$ 0.05). At the later time point, serum insulin like growth factor binding protein-3 levels were significantly higher in the $<40 \%$ TBSA and 40-59\% TBSA burn groups when compared with the other two burn groups $(P<0.05)$ (Figure 4). Serum growth hormone markedly decreased in burns of $>40 \%$ TBSA and was significantly higher in burns of $<40 \%$ TBSA $(P<$ 0.05 ) (Figure 4). Serum insulin was significantly increased in large burns immediately after burn compared with the small burns $(P<0.05)$ (Figure 4$)$. Insulin decreased over time in all groups.

Urine cortisol was increased during the acute stay in all four groups. The urine cortisol level, however, was significantly lower in the $<40 \%$ TBSA burn group when compared with the other three groups $(P<0.05)$ (Figure 4$)$.

In terms of serum cytokines, we found that only six cytokines were significantly and possibly clinically relevantly affected. Serum IL-8, TNF, IL-6, IL-12p70, monocyte chemoattractant protein-1 and granulocyte-macrophage colony-stimulating factor were significantly increased in the large burns. In general, there was a notion that the smaller the burn size, the lower the cytokine concentration - indicating a relationship between burn size and cytokine expression (Figure 5).

Multiple cytokines, such as IL-1 $\beta$, macrophage inflammatory protein-1 $\beta$, IFN $\gamma, \mathrm{IL}-10$, granulocyte colony-stimulating factor,
Figure 2

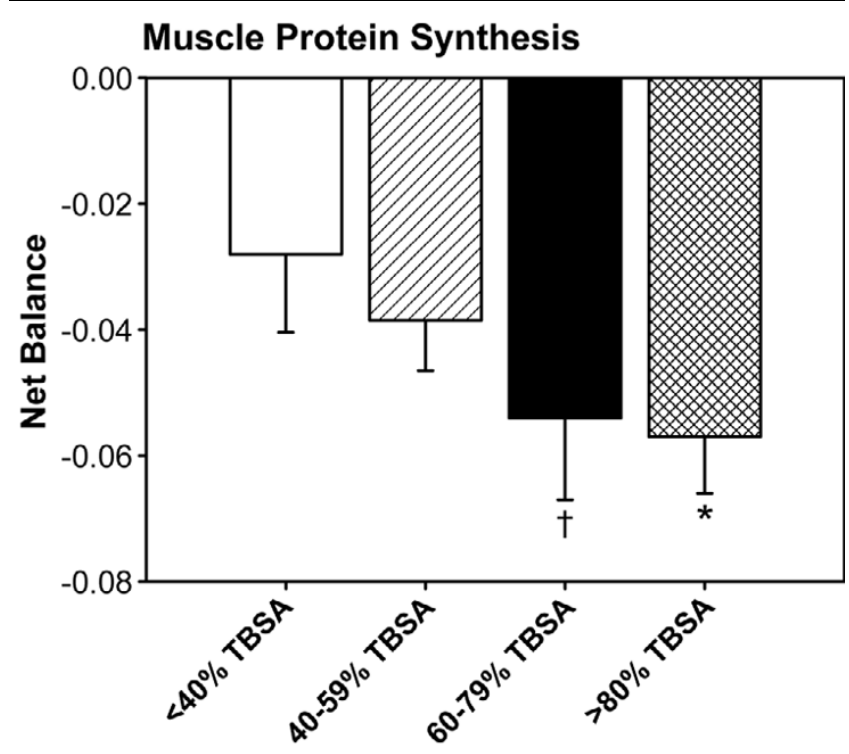

Peripheral muscle protein net balance during acute hospitalization. All burned children had a negative net protein balance in skeletal muscle. The greatest muscle protein loss was found in children with 60-79\% and $>80 \%$ total body surface area (TBSA) burn. ${ }^{\star}$ Significant difference between $>80 \%$ TBSA burn group versus $<40 \%$ TBSA burn group, $P<$ 0.05 .

IL-17, IL-13, IL-7, IL-5, IL-4, and IL-2, were affected by the burn but not by the burn size. Cytokines increased with the injury and decreased over time. These cytokines showed no significant difference between the burn size groups, but presented significant changes over time.

\section{Liver and cardiac changes}

Analysis of the cardiac output, the predicted cardiac output, the stroke volume, the predicted stroke volume, the heart rate, the predicted heart rate, the cardiac index, and the central venous pressure showed differences between groups. The cardiac output and the predicted cardiac output were significantly decreased in the burns $>80 \%$ TBSA $(P<0.05)$, while there was no difference between the three other burn groups (Figure 6). The predicted stroke volume was also significantly decreased in the $>80 \%$ TBSA burn group when compared with the other three groups $(P<0.05)$ (Figure 6). There were, however, no differences between groups in the heart rate, the predicted heart rate, the cardiac index, and the central venous pressure (Figure 6).

Immediately after burn, the liver size increased by almost $100 \%$ in all groups (Figure 7). While the liver in burns of $>60 \%$ TBSA further increased in size to $150 \%$, the liver size in burns of $<40 \%$ TBSA decreased rapidly $(P<0.05)$ (Figure 7$)$. There was no significant difference in liver size increase between burns of $40-59 \%$ TBSA, of $60-79 \%$ TBSA, and of $>80 \%$ TBSA (Figure 7). 
Figure 3

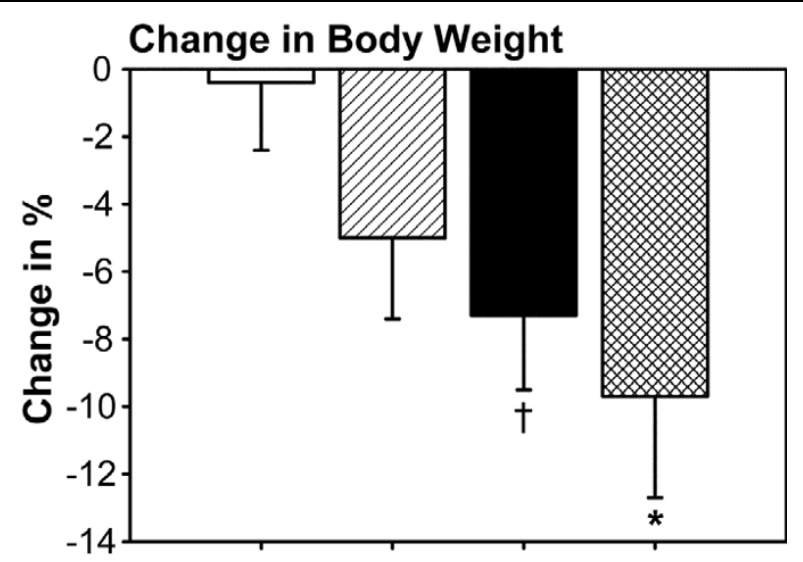

Change in Lean Body Mass

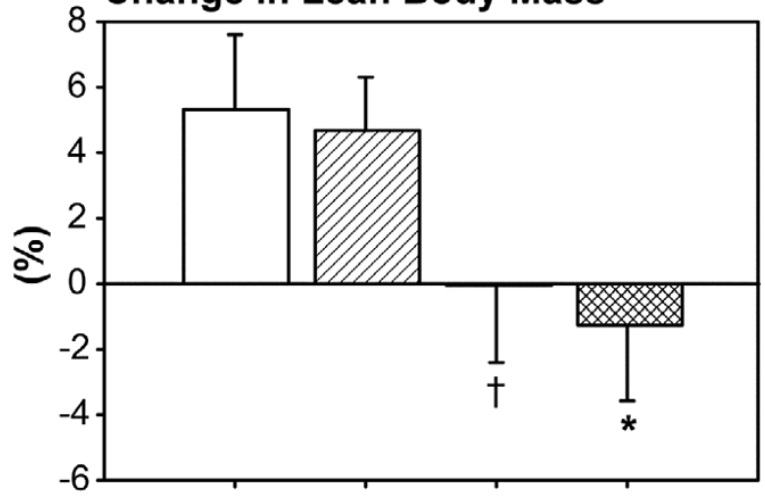

Time Postburn Change in Total Bone Mineral Content

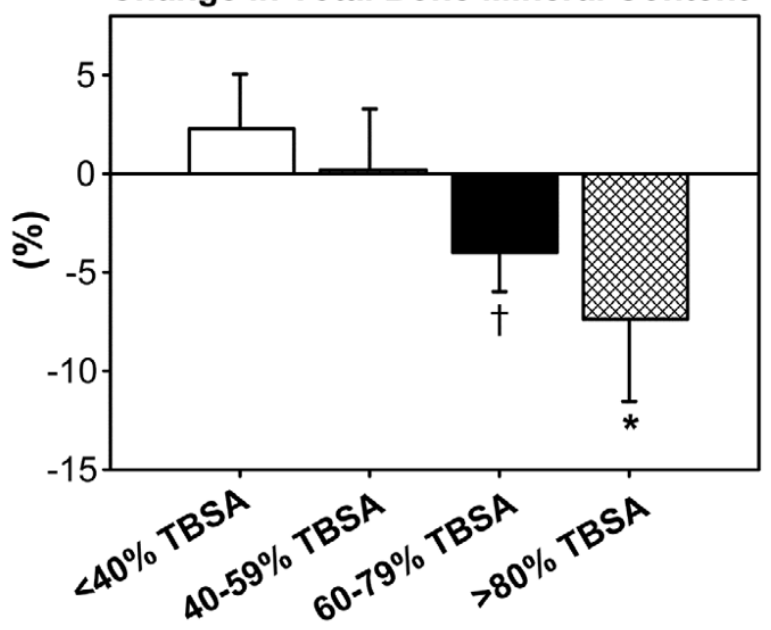

Change in body composition from admission to discharge. Children with $>80 \%$ total body surface area (TBSA) burn lost the most body weight, lean body mass and bone mineral content compared with the other groups. The 60-79\% TBSA burn group showed a significant loss in body weight, lean body mass and bone mineral content that was significant compared with the $<40 \%$ and $40-59 \%$ TBSA groups. The $<40 \%$ and $40-59 \%$ TBSA burn groups had almost no loss in body weight, lean body mass and bone mineral content. *Significant difference between $>80 \%$ TBSA burn group versus $<40 \%$ TBSA burn group, $P<0.05$. 'Significant difference $60-79 \%$ TBSA burn group versus $<40 \%$ TBSA burn group, $P<0.05$.

\section{Discussion}

The metabolic rate in burns is extremely high, and energy requirements are immense and are met by the mobilization of proteins and amino acids [5]. As a consequence, the structure and function of essential organs such as skeletal muscle, skin, immune system, and cellular membrane transport functions are compromised [17-19]. Catecholamines involved in the hypermetabolic response to burn injury are released from sympathetic nerve ends and the adrenal medulla, and are raised two-fold to 10-fold in proportion to the burn size [2023]. In the present study, by comparing four different burn sizes, we showed that an increase in burn size is associated with increased hypermetabolism, with persistent inflammation, with catabolism, with changes in body composition, with increased stress hormone production, and with organ dysfunction. Whether the correlation between these phenomena and burn size is linear was not determined, but we clearly observed these pathophysiologic changes with increased burn size.

Large burns cause a marked increase in inflammation and catecholamines, which leads to an increased metabolic rate. In the present study, we found that smaller burn injuries demonstrated decreased catecholamine and stress hormone levels that were associated with decreased hypermetabolism and catabolism. Smaller burns had significantly decreased and shortened predicted REE, body weight loss, and net muscle protein balance when compared with the larger burns. Further indicators that smaller burns are less hypermetabolic than larger burns are decreased acute stress hormones and increased anabolic hormones. Catecholamines, cytokines and proinflammatory mediators are known to block, and therefore decrease, endogenous anabolic agents via cellular mediators $[24,25]$.

We showed in the present study that patients with large burns demonstrate a different cytokine expression profile compared with patients with smaller burn injuries. Patients with burns over $80 \%$ TBSA had persistent and marked increased levels of IL-8, IL-6, monocyte chemoattractant protein-1 and TNF. All of these cytokines are proinflammatory and enhance catabolism and hypermetabolism via hyperinflammation. We therefore suggest that these high levels of proinflammatory cell mediator trigger and enhance the hypermetabolic response with increased stress hormones and protein catabolism. On the other hand, lower inflammation and hypermetabolism was associated with higher endogenous anabolic hormone levels. Patients with smaller burns had lower inflammatory marker and stress hormones, which was associated with higher IGF-I, insulin like growth factor binding protein-3 and growth hormone levels. IGF-I was shown to exert anabolic effects on skeletal muscle protein synthesis, to attenuate the hepatic acute phase response, and to improve dermal and epidermal regeneration [26-30]. Furthermore, decreased growth hormone and 

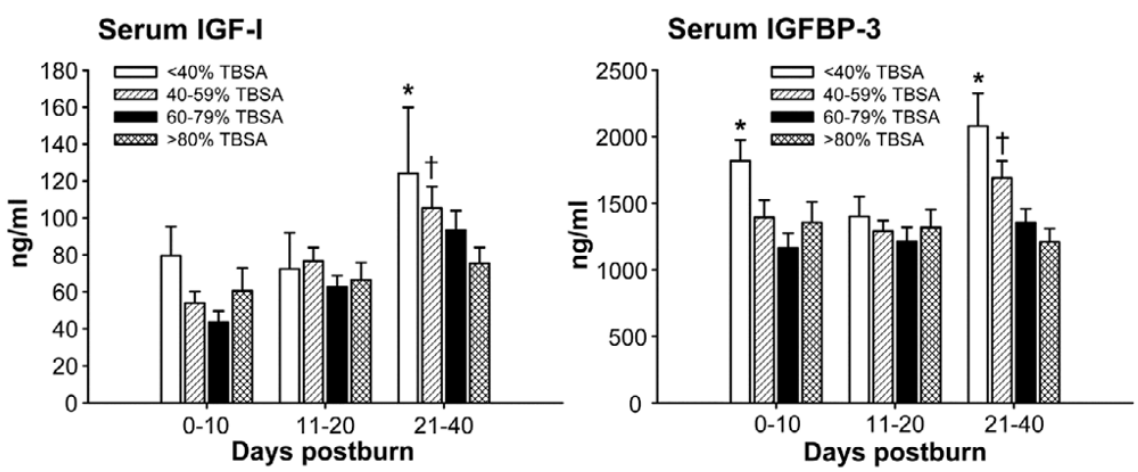

Serum GH
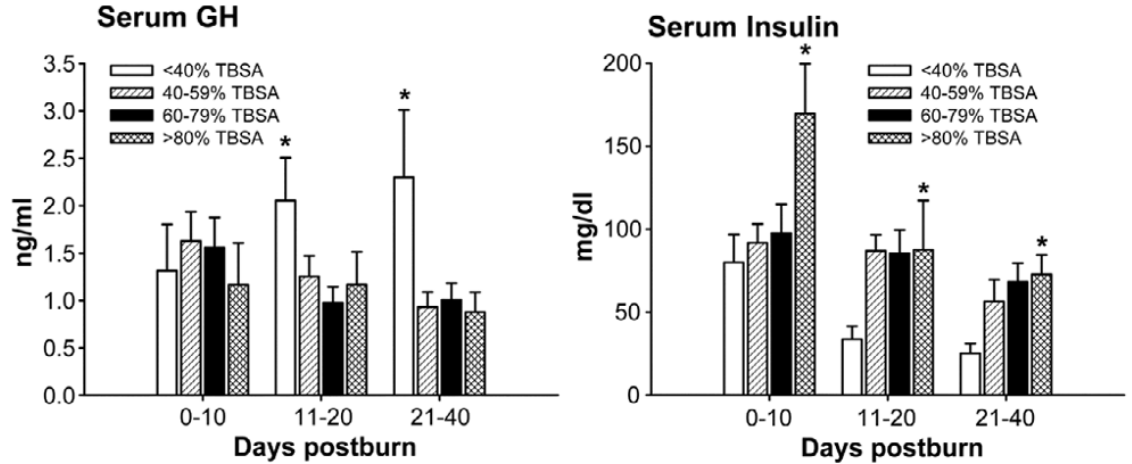

Urine Cortisol

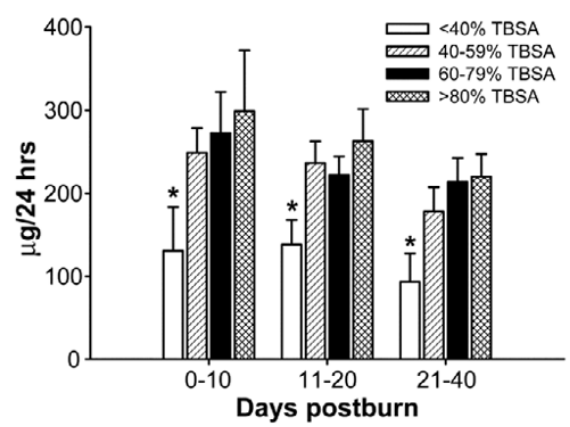

Serum protein concentrations during acute hospitalization at multiple time points. Serum insulin growth factor-I (IGF-I) was significantly increased in the $<40 \%$ and $40-59 \%$ total body surface area (TBSA) burn groups compared with the $60-79 \%$ and $>80 \%$ TBSA burn groups $21-40$ days postburn. At the later time point, serum insulin like growth factor binding protein-3 (IGFBP-3) was significantly higher in the $<40 \%$ and $40-59 \%$ TBSA burn groups when compared with the other two burn groups. Serum growth hormone $(\mathrm{GH})$ markedly decreased in burns of $>40 \%$ TBSA and was significantly higher in burns of $<40 \%$ TBSA. Serum insulin was significantly increased in large burns immediately after burn compared with the small burns. Urine cortisol was increased during the acute stay in all four groups. Urine cortisol, however, was significantly lower in the $<40 \%$ TBSA burn group when compared with the other three groups. *Significant difference between $>80 \%$ TBSA burn group versus $<40 \%$ TBSA burn group, $P<$ 0.05 . 'Significant difference $60-79 \%$ TBSA burn group versus $<40 \%$ TBSA burn group, $P<0.05$. Normal levels: IGF-I, $220-260$ pg/ml; IGFBP-3, $3,800-4,200 \mathrm{pg} / \mathrm{ml} ; \mathrm{GH}, 6 \mathrm{pg} / \mathrm{ml}$; insulin, $10-25 \mathrm{mg} / \mathrm{dl}$; and urine cortisol, 20-45 $\mu \mathrm{g} / 24$ hours.

IGF-I levels lead to a deficit in transmembrane amino acid transport and a compromised immune system [31-35].

The glucose kinetics in severely burned patients is almost always abnormal [36,37]. Glucose utilization in burned patients is almost entirely through inefficient anaerobic mechanisms, as characterized by increased lactate production, which accounts for increased glucose consumption $[22,23,38]$. Glucose production, particularly from alanine, is elevated in almost all patients with severe burn [5]. The increased gluconeogenesis from amino acids renders these amino acids unavailable for reincorporation into body protein. Nitrogen is excreted, primarily in urea, thus contributing to the progressive depletion of body protein stores. Plasma insulin levels usually remain normal or slightly elevated in burn patients $[39,40]$. The fact that the basal rate of glucose production is elevated despite normal or elevated plasma insulin levels indicates hepatic insulin resistance, since under normal conditions elevated serum insulin would lower the rate of glucose production [38-40]. Furthermore, the plasma glucose 

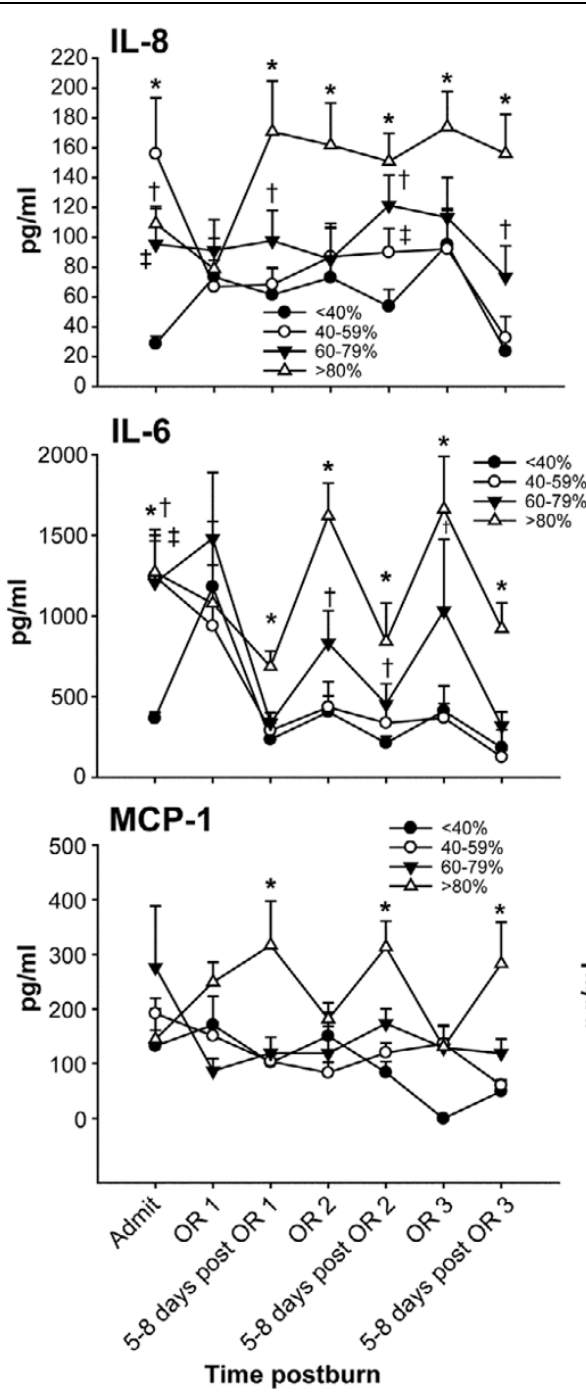
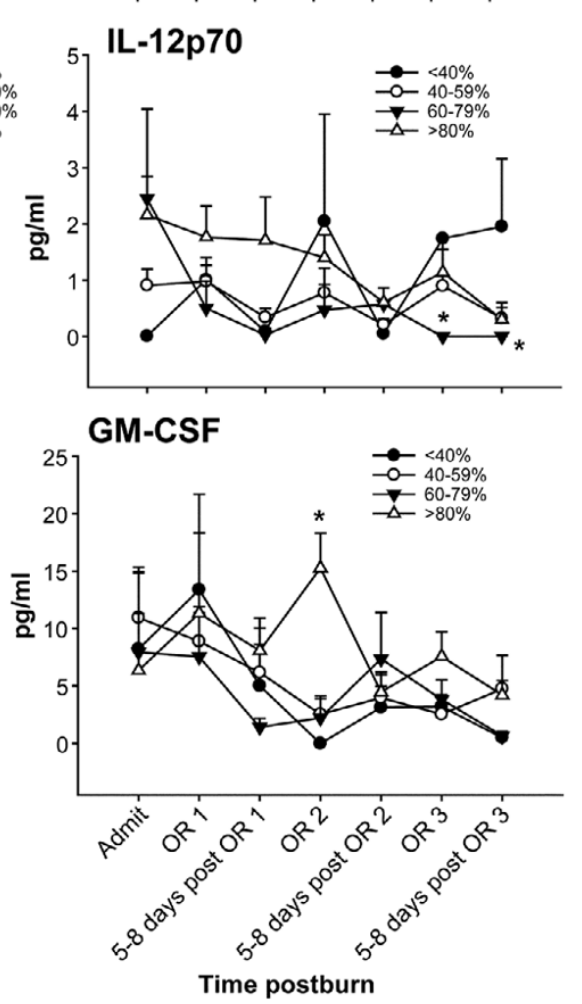

Cytokine concentrations in relation to acute hospitalization. Cytokines were measured at admission (Admit), at first surgery (OR1), at 5-8 days postsurgery 1 , at second surgery (OR2), at 5-8 days postsurgery 2 , at third surgery (OR3), and again at 5-8 days postsurgery 3 . Six cytokines out of 17 measured were significantly different between different burn sizes. Serum IL-8, TNF, IL-6, IL-12p70, monocyte chemoattractant protein-1 (MCP-1) and granulocyte-macrophage colony-stimulating factor (GM-CSF) were significantly increased in the large burns compared with the other three groups. *Significant difference between $>80 \%$ TBSA burn group versus $60-79 \%, 40-59 \%$ and $<40 \%$ TBSA burn groups, $P<0.05$. ${ }^{+}$Significant difference between $60-79 \%$ TBSA burn group versus $40-79 \%$ and $<40 \%$ TBSA burn groups, $P<0.05$. ${ }^{\circ}$ Significant difference between $40-59 \%$ TBSA burn group versus $<40 \%$ TBSA burn group, $P<0.05$. Normal levels: IL-8, $7.6 \pm 3.9 \mathrm{pg} / \mathrm{ml} ; \mathrm{TNF}, 0.7 \pm 0.07 \mathrm{pg} / \mathrm{ml} ; \mathrm{lL}-6,8.7 \pm 4.9 \mathrm{pg} / \mathrm{ml} ; \mathrm{IL}-$ $12 \mathrm{p} 70,0 \pm 0 \mathrm{pg} / \mathrm{ml} ; \mathrm{MCP}-1,42 \pm 5 \mathrm{pg} / \mathrm{ml}$; and GM-CSF, $0 \pm 0 \mathrm{pg} / \mathrm{ml}$.

concentration is frequently increased, which would normally directly inhibit glucose production [41,42]. As hyperglycemia is associated with increased mortality in critically ill patients $[43,44]$ and worsens the outcome in severely burned patients $[41,42]$, we determined serum insulin levels in our four burn groups. We found that insulin levels were significantly increased in the $>80 \%$ TBSA burn group and were lower in the smaller burn groups. This indicates that with the increased severity of the burn injury insulin resistance increases and more insulin needs to be synthesized to maintain normoglycemia.
Another striking finding of this study was the cardiac function. Burn patients with burns of $>80 \%$ TBSA demonstrated significantly decreased cardiac output and percentage predicted cardiac output. Furthermore, these patients had significantly decreased percentage predicted stroke volumes and cardiac index when compared with the other groups. There were no differences in the heart rate, the predicted heart rate, and the central venous pressure between groups. As the preload was not significantly different between groups, we therefore suggest that a severe burn over $80 \%$ TBSA causes a marked myocardial depression. A myocardial depression associated with a severe burn injury has been shown in several studies 

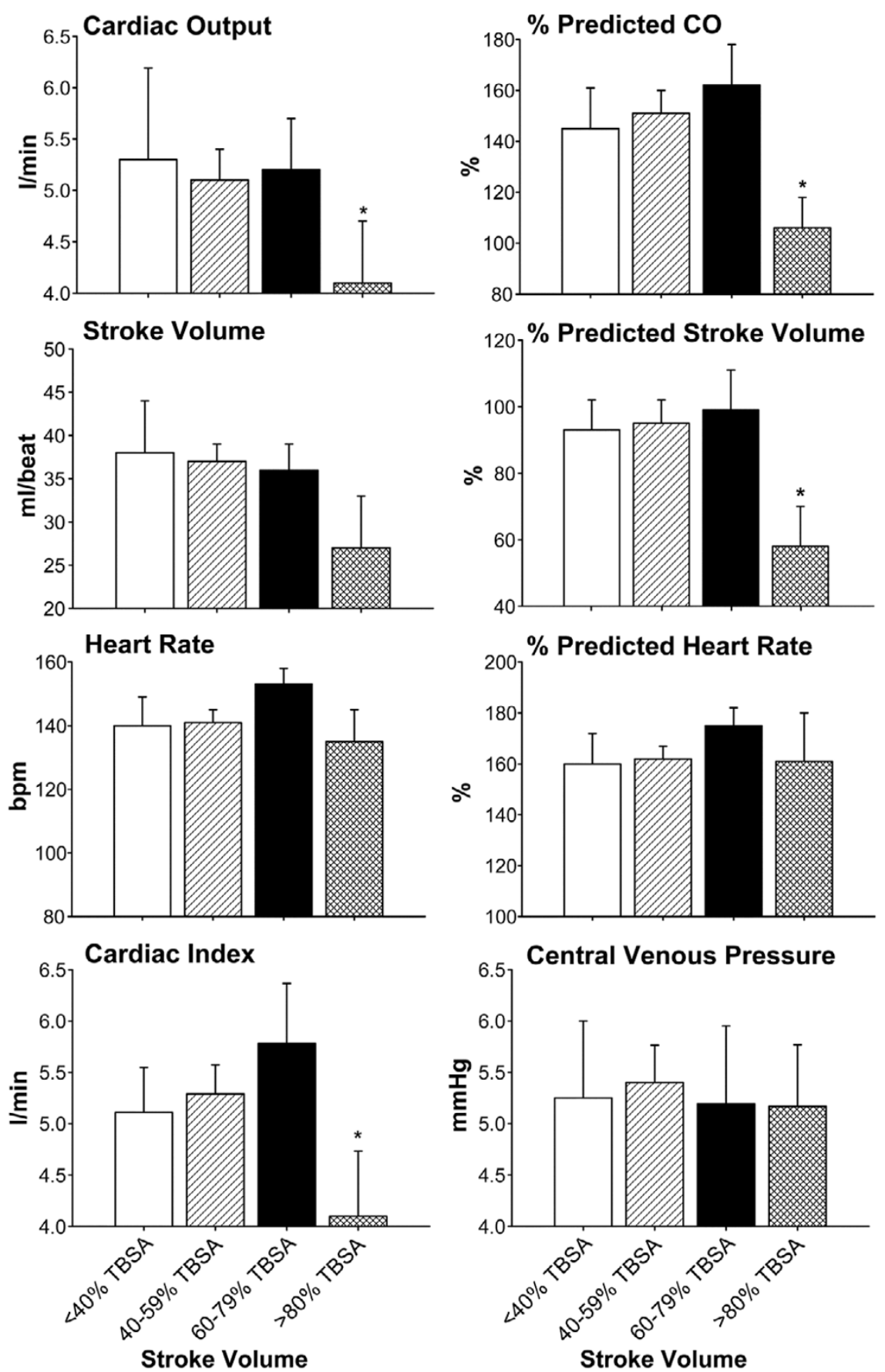

Cardiac function within 96 hours after hospital admission. The cardiac output and the predicted cardiac output (predicted CO) were significantly decreased in burns of $>80 \%$ total body surface area (TBSA), while there was no difference between the three other burn groups. The predicted stroke volume was significantly decreased in the $>80 \%$ TBSA burn group when compared with the other three groups. There were, however, no differences between groups in the heart rate, predicted heart rate, cardiac index, and central venous pressure. * Significant difference between $>80 \%$ TBSA burn group versus $60-79 \%, 40-59 \%$ and $<40 \%$ TBSA burn groups, $P<0.05$.

[45-47]. These data demonstrate that myocardial depression plays an important role during the postburn response and that modulation of myocardial depression may improve clinical outcome. The hypothesis that myocardial dysfunction may be one of the main contributors to mortality in large burns was confirmed in a recent retrospective autopsy study [10]. 
Figure 7

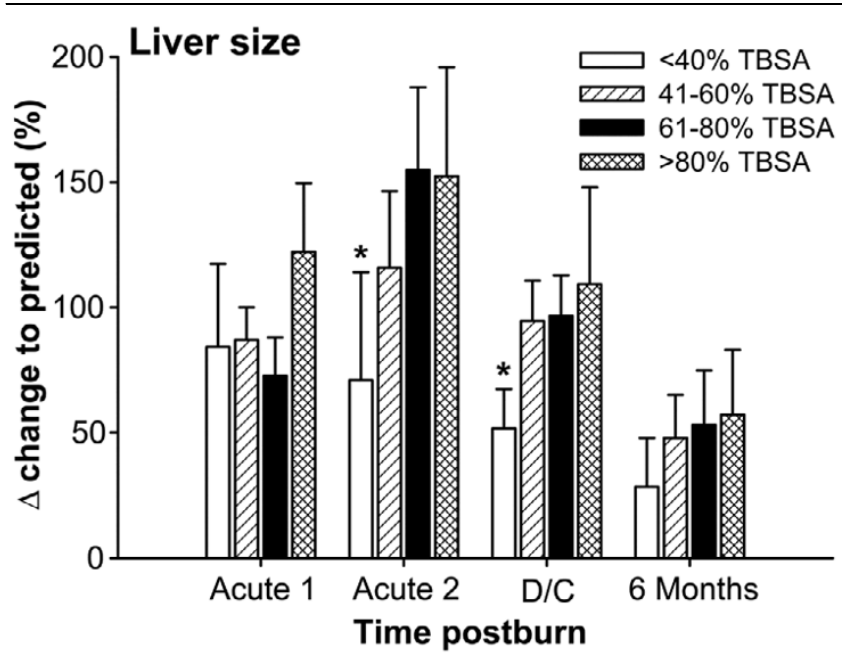

Liver size increased by almost $100 \%$ immediately after burn in all groups. The liver size was significantly smaller in $<40 \%$ total body surface area (TBSA) burns compared with the other groups. *Significant difference between $<40 \%$ TBSA burn group versus $40-59 \%, 60-$ $79 \%$ and $>80 \%$ TBSA burn groups, $P<0.05$. Measurements were performed at week 1 (Acute 1), at weeks 2-4 (Acute 2), at discharge (D/C), and at 6 months postburn.

\section{Conclusion}

Based on our findings, we suggest that a burn injury involving more than $80 \%$ of the total body surface causes marked and prolonged inflammation, marked increases in hypermetabolism, catabolism and cardiac dysfunction, and, subsequently, higher incidences of infection, sepsis and death. Treatment should focus on several aspects of the pathophysiologic events postburn, such as treatment of the inflammatory response, insulin resistance, hypermetabolism, catabolism, and cardiac dysfunction.

\section{Competing interests}

The authors declare that they have no competing interests.

\section{Authors' contributions}

MGJ designed the study, gathered data, conducted the statistics and wrote the manuscript. RPM collected data, reviewed the statistical analysis and reviewed the manuscript. CCF performed experiments to obtain data, conducted the statistical analysis, and helped to write the manuscript. WBN helped to collect data and write the manuscript. GAK helped to obtain data, performed and established methods to obtain serum analyses, and reviewed the manuscript. GGG collected data, reviewed the statistical analysis and reviewed the manuscript. $\mathrm{DNH}$ gathered data, reviewed the analysis, and helped to write the manuscript.

\section{Acknowledgements}

This study was supported by the American Surgical Association Foundation, by the Shriners Hospitals for Children 8660,8760 , and 9145 , by the National Institutes of Health R01-GM56687, T32 GM008256, and

\section{Key messages}

- The inflammatory and metabolic response is dependent on the burn size.

- Under 60\% TBSA burn, the hypermetabolic and inflammatory response is less pronounced.

- Between $60 \%$ and $80 \%$ TBSA burn, patients are severely hypermetabolic and hyperinflammatory, but they do not undergo cardiac dysfunction.

- Cardiac dysfunction only occurs in burns over $80 \%$ TBSA, and patients undergo a drastic inflammatory and hypermetabolic response.

- Patients should be referred to a major burn center with a burn size over 40-60\% TBSA; antinflammatory and anabolic treatment should be initiated for burn sizes of 40-60\% TBSA; however, cardiac monitoring and treatment must be initiated in burns over $80 \%$ TBSA.

- Cardiac dysfunction appears one of the major contributing factors for mortality, and the treatment of cardiac dysfunction may improve survival.

P50 GM60338, and by the National Institute on Disability and Rehabilitation Research $\mathrm{H} 133 \mathrm{~A} 020102$.

\section{References}

1. Finnerty CC, Herndon DN, Przkora R, Pereira CT, Oliveira HM, Queiroz DM, Rocha AM, Jeschke MG: Cytokine expression profile over time in severely burned pediatric patients. Shock 2006, 26:13-19.

2. Herndon DN, Tompkins RG: Support of the metabolic response to burn injury. Lancet 2004, 363:1895-1902.

3. Pereira C, Murphy K, Jeschke M, Herndon DN: Post burn muscle wasting and the effects of treatments. Int J Biochem Cell Biol 2005, 37:1948-1961.

4. Przkora R, Barrow RE, Jeschke MG, Suman OE, Celis M, Sanford AP, Chinkes DL, Mlcak RP, Herndon DN: Body composition changes with time in pediatric burn patients. J Trauma 2006 60:968-971.

5. Rennie MJ: Muscle protein turnover and the wasting due to injury and disease. Br Med Bull 1985, 41:257-264.

6. Arnold J, Campbell IT, Samuels TA, Devlin JC, Green CJ, Hipkin LJ, MacDonald IA, Scrimgeour CM, Smith K, Rennie MJ: Increased whole body protein breakdown predominates over increased whole body protein synthesis in multiple organ failure. Clin Sci (Lond) 1993, 84:655-661.

7. Biolo G, Tipton KD, Klein S, Wolfe RR: An abundant supply of amino acids enhances the metabolic effect of exercise on muscle protein. Am J Physiol 1997, 273(1 Pt 1):E122-E1 29.

8. Biolo G, Toigo G, Ciocchi B, Situlin R, Iscra F, Gullo A, Guarnieri G: Metabolic response to injury and sepsis: changes in protein metabolism. Nutrition 1997, 13(9 Suppl):52S-57S.

9. World Health Organization: A graphical overview of the global burden of injuries. In The Injury Chart Book Geneva: World Health Organization; 2002:29.

10. Pereira CT, Barrow RE, Sterns AM, Hawkins HK, Kimbrough CW, Jeschke MG, Lee JO, Sanford AP, Herndon DN: Age-dependent differences in survival after severe burns: a unicentric review of 1,674 patients and 179 autopsies over 15 years. $J \mathrm{Am}$ Coll Surg 2006, 202:536-548.

11. Weir JB: New methods for calculating metabolic rate with special reference to protein metabolism. J Physiol 1949, 109:1-9.

12. Harris J, Benedict F: A Biometric Study of Basal Metabolism in Man Carnegie Institution; 1919. 
13. Wolfe RR, Chinkes DL: Principles and Practice of Kinetic Analysis NJ: Wiley-Liss; 2005.

14. Hart DW, Wolf SE, Chinkes DL, Gore DC, Mlcak RP, Beauford RB, Obeng MK, Lal S, Gold WF, Wolfe RR, et al:: Determinants of skeletal muscle catabolism after severe burn. Ann Surg 2000, 232:455-465.

15. Hart DW, Wolf SE, Herndon DN, Chinkes DL, Lal SO, Obeng MK, Beauford RB, Mlcak RR: Energy expenditure and caloric balance after burn: increased feeding leads to fat rather than lean mass accretion. Ann Surg 2002, 235:152-161.

16. Hart DW, Wolf SE, Zhang XJ, Chinkes DL, Buffalo MC, Matin SI, DebRoy MA, Wolfe RR, Herndon DN: Efficacy of a high-carbohydrate diet in catabolic illness. Crit Care Med 2001, 29:1318-1324.

17. Biolo G, Fleming RY, Maggi SP, Wolfe RR: Transmembrane transport and intracellular kinetics of amino acids in human skeletal muscle. Am J Physiol 1995, 268(1 Pt 1):E75-E84.

18. Biolo G, Maggi SP, Williams BD, Tipton KD, Wolfe RR: Increased rates of muscle protein turnover and amino acid transport after resistance exercise in humans. Am J Physio/ 1995, 268(3 Pt 1):E514-E520.

19. Biolo G, Wolfe RR: Relationship between plasma amino acid kinetics and tissue protein synthesis and breakdown. Proceedings of the IFAC Symposium on Modeling Control in Biological Systems 1994:358-359.

20. Goodall M, Stone C, Haynes BW Jr: Urinary output of adrenaline and noradrenaline in severe thermal burns. Ann Surg 1957, 145:479-487.

21. Goodall MG: Sympathetic nerve and adrenal medullary response to thermal burn. Clinical analysis of adrenaline and noradrenaline depletion. Am Surg 1966, 32:448-452.

22. Wilmore DW: Hormonal responses and their effect on metabolism. Surg Clin North Am 1976, 56:999-1018.

23. Wilmore DW, Long JM, Mason AD Jr, Skreen RW, Pruitt BA Jr: Catecholamines: mediator of the hypermetabolic response to thermal injury. Ann Surg 1974, 180:653-669.

24. Delhanty PJ: Interleukin-1 beta suppresses growth hormoneinduced acid-labile subunit mRNA levels and secretion in primary hepatocytes. Biochem Biophys Res Commun 1998, 243:269-272.

25. Wang G, Lee HM, Englander E, Greeley GH Jr: Ghrelin - not just another stomach hormone. Regul Pept 2002, 105:75-81.

26. Debroy MA, Wolf SE, Zhang XJ, Chinkes DL, Ferrando AA, Wolfe RR, Herndon DN: Anabolic effects of insulin-like growth factor in combination with insulin-like growth factor binding protein3 in severely burned adults. J Trauma 1999, 47:904-911.

27. Herndon DN, Ramzy PI, DebRoy MA, Zheng M, Ferrando AA, Chinkes DL, Barret JP, Wolfe RR, Wolf SE: Muscle protein catabolism after severe burn: effects of IGF-1/IGFBP-3 treatment. Ann Surg 1999, 229:713-722.

28. Jeschke MG, Barrow RE, Suzuki F, Rai J, Benjamin D, Herndon DN: IGF-I/IGFBP-3 equilibrates ratios of pro- to anti-inflammatory cytokines, which are predictors for organ function in severely burned pediatric patients. Mol Med 2002, 8:238-246.

29. Jeschke MG, Herndon DN, Barrow RE: Insulin-like growth factor $I$ in combination with insulin-like growth factor binding protein 3 affects the hepatic acute phase response and hepatic morphology in thermally injured rats. Ann Surg 2000, 231:408-416.

30. Jeschke MG, Herndon DN, Vita R, Traber DL, Jauch KW, Barrow RE: IGF-I/BP-3 administration preserves hepatic homeostasis after thermal injury which is associated with increases in no and hepatic NF-kappa B. Shock 2001, 16:373-379.

31. Binoux M: The IGF system in metabolism regulation. Diabete Metab 1995, 21:330-337.

32. Ericsson A, Hamark B, Jansson N, Johansson BR, Powell TL, Jansson $T$ : Hormonal regulation of glucose and system $A$ amino acid transport in first trimester placental villous fragments. Am J Physiol Regul Integr Comp Physiol 2005, 288:R656-R662.

33. Inoue $Y$, Copeland EM, Souba WW: Growth hormone enhances amino acid uptake by the human small intestine. Ann Surg 1994, 219:715-724.

34. Inoue $Y$ Fallon W Souba WW: Growth hormone attenuates $\mathrm{Na}(+)$-dependent hepatic amino acid transport in endotoxemic rats. J Trauma 1993, 35:605-612.
35. Pacitti AJ, Inoue Y, Plumley DA, Copeland EM, Souba WW: Growth hormone regulates amino acid transport in human and rat liver. Ann Surg 1992, 216:353-362.

36. Demling RH, DeSanti L: Effect of a hypocaloric diet, increased protein intake and resistance training on lean mass gains and fat mass loss in overweight police officers. Ann Nutr Metab 2000, 44:21-29.

37. Demling $\mathrm{RH}$, Seigne $\mathrm{P}$ : Metabolic management of patients with severe burns. World J Surg 2000, 24:673-680.

38. Gore DC, Ferrando A, Barnett J, Wolf SE, Desai M, Herndon DN, Goodwin C, Wolfe RR: Influence of glucose kinetics on plasma lactate concentration and energy expenditure in severely burned patients. J Trauma 2000, 49:673-678.

39. Wolfe RR, Nadel ER, Shaw JH, Stephenson LA, Wolfe MH: Role of changes in insulin and glucagon in glucose homeostasis in exercise. J Clin Invest 1986, 77:900-907.

40. Wolfe RR, Shaw JH, Jahoor F, Herndon DN, Wolfe MH: Response to glucose infusion in humans: role of changes in insulin concentration. Am J Physiol 1986, 250(3 Pt 1):E306-E311.

41. Gore DC, Chinkes D, Heggers J, Herndon DN, Wolf SE, Desai M: Association of hyperglycemia with increased mortality after severe burn injury. J Trauma 2001, 51:540-544.

42. Gore DC, Chinkes DL, Hart DW, Wolf SE, Herndon DN, Sanford AP: Hyperglycemia exacerbates muscle protein catabolism in burn-injured patients. Crit Care Med 2002, 30:2438-2442.

43. Van den Berghe G, Wilmer A, Hermans G, Meersseman W, Wouters PJ, Milants I, Van Wijngaerden E, Bobbaers $\mathrm{H}$, Bouillon R: Intensive insulin therapy in the medical ICU. N Engl J Med 2006, 354:449-461.

44. van den Berghe $G$, Wouters $P$, Weekers F, Verwaest C, Bruyninckx F, Schetz M, Vlasselaers D, Ferdinande P, Lauwers P, Bouillon R: Intensive insulin therapy in the critically ill patients. $N$ Engl J Med 2001, 345:1359-1367.

45. Sambol JT, White J, Horton JW, Deitch EA: Burn-induced impairment of cardiac contractile function is due to gut-derived factors transported in mesenteric lymph. Shock 2002, 18:272-276.

46. Willis MS, Carlson DL, Dimaio JM, White MD, White DJ, Adams GAt, Horton JW, Giroir BP: Macrophage migration inhibitory factor mediates late cardiac dysfunction after burn injury. $A m$ $J$ Physiol Heart Circ Physiol 2005, 288:H795-H804.

47. Yang J, Yang Z, Chen F: Myocardial contractile and calcium transport function after severe burn injury. Zhonghua Zheng Xing Shao Shang Wai Ke Za Zhi 1998, 14:211-213. 